\title{
TECHNOLOGY AND CHALLENGES OF LINEAR COLLIDERS
}

\author{
R. Brinkmann \\ DESY, Notkestr. 85, D-22603 Hamburg, Germany
}

\section{Abstract}

In this talk I'll briefly review the design challenges of a high energy, high luminosity e+e- linear collider and discuss the layout and performance of various technical options. I'll then present the status of the R\&D effort and review the performance of the integrated system tests now underway at several laboratories.

\section{INTRODUCTION}

With the approved LHC project at CERN the energy frontier of Particle Physics will be pushed into new territory, almost an order of magnitude higher than the reach of presently operating collider facilities. In the international community of High Energy Physics there is widespread agreement that an e+e- Linear Collider of 500 $\mathrm{GeV}$ center-of-mass energy (upgradable to about $1 \mathrm{TeV}$ ) should be the next project to continue the complementary research with hadron and lepton machines, which has proven extremely important and fruitful in the past. Studies towards a next generation Linear Collider are being pursued at several High Energy Physics Laboratories [1]. At present, one can distinguish three different approaches for such a future linear accelerator. The conventional concept of NLC/JLC (SLAC/KEK study) using travelling wave copper structures driven by high peak-power klystrons, similar to the SLC, but at Xband frequency (four times the $3 \mathrm{GHz}$ frequency of the SLAC linac) in order to reach higher gradients. Second, the CLIC study (CERN) still using similar structures but at even higher frequency and replacing the klystrons by a relativistic high power drive beam from which the rf power is extracted. The third concept, pursued by a broad international collaboration centered at DESY, is the TESLA approach of a superconducting linear accelerator. In the following, a brief overview of the different linear collider concepts will be given and the status of the technical developments will be summarised.

\section{MACHINE PARAMETERS}

The key parameter (in addition to the center-of-mass energy) of a linear collider is the luminosity

$$
L=\frac{f_{r e p} n_{b} N_{e}^{2}}{4 \pi \sigma_{x} \sigma_{y}}
$$

where $f_{\text {rep }}$ denotes the pulse repetition frequency of the linear accelerator, $n_{b}$ the number of bunches per pulse, $N_{e}$ the bunch charge and $\sigma_{\mathrm{x}(\mathrm{y})}$ the horizontal (vertical) beam size at the interaction point (IP for short). It can be shown that the luminosity is essentially limited by the AC-power $P_{A C}$ taken from the grid, the overall efficiency $\eta$ of converting $\mathrm{P}_{\mathrm{AC}}$ into beam power, the energy loss $\delta_{\mathrm{B}}$ due to beamstrahlung at the IP and the normalized vertical beam emittance $\gamma \varepsilon_{\mathrm{y}}$ :

$$
L \propto \eta P_{A C} \sqrt{\frac{\delta_{B}}{\gamma \varepsilon_{y}}}
$$

The power consumption has to remain within reasonable limits and the beamstrahlung has to be limited to a few percent of the beam energy in order to maintain good energy resolution and small background in the interaction region. Thus the only remaining free parameters to optimize the machine performance in terms of the luminosity are the efficiency and the emittance.

An overview of the basic parameters for the X-band, CLIC and TESLA $500 \mathrm{GeV}$ designs is given in Table 1. A common feature of all approaches is operation with long trains of bunches per rf-pulse, necessary to achieve a high rf-to-beam power transfer efficiency. Due to the extremely low power losses in the walls of the superconducting resonators, the pulse length in TESLA can be several orders of magnitude larger than in a conventional accelerator, resulting in a low rf-peak power requirement. Furthermore, in that case the time structure of the beam with large spacing between bunches allows to apply energy and orbit feedback [2] on a bunch-to-bunch basis, thus making the superconducting linac almost immune with respect to pulse-to-pulse drift and jitter effects. The spot size at the IP is two orders of magnitude below what is routinely achieved at the SLC. In addition to a special magnet lattice for beam size demagnification (Final Focus System, see below), good control of emittance dilution in the linac is indispensable to achieve this ambitious goal [3-5]. Wakefields excited by the beam in the accelerating structures are the main cause of emittance blow-up. Since wakefields are strongly dependent on the linac frequency (the transverse wakefield scales approximately proportional to the $3^{\text {rd }}$ power of the frequency), it is no surprise that the alignment tolerances and the needs for frequent orbit optimization and tuning are more stringent for the highfrequency conventional linacs than for TESLA (e.g. for the structure alignment $10-15 \mu \mathrm{m}$ as compared to 
$0.5 \mathrm{~mm})$. The realistically achievable beam emittance at the IP is thus lower for TESLA, which, together with the high efficiency, leads to a higher design value of the luminosity. On the other hand, the site length for the Xband and two-beam machines can be shorter thanks to the higher accelerating gradient.

Table 1: Main parameters of the TESLA, X-Band and Two-beam linear colliders at $500 \mathrm{GeV}$ center-of-mass energy.

\begin{tabular}{|l|l|l|l|}
\hline & TESLA & X-band & CLIC \\
\hline Frequency $[\mathrm{GHz}]$ & 1.3 & 11.4 & 30 \\
\hline Gradient $[\mathrm{MV} / \mathrm{m}]$ & 22 & 57 & 100 \\
\hline AC power $[\mathrm{MW}]$ & 95 & 99 & 68 \\
\hline Efficiency $\eta[\%]$ & 23 & 10 & 14 \\
\hline Emittance $\gamma \varepsilon_{x, y}[\mu \mathrm{m}]$ & $10,0.03$ & $5,0.1$ & $2,0.1$ \\
\hline Spot at IP $\sigma_{x, y}[\mathrm{~nm}]$ & 553,5 & $335,4.5$ & $196,4.5$ \\
\hline Beamstrlng. $\delta_{\mathrm{B}}[\%]$ & 3 & 4 & 3.6 \\
\hline Lumin. $\left[10^{\left.3 \mathrm{~cm}^{-2} \mathrm{~s}^{-1}\right]}\right.$ & 31 & 7 & 5 \\
\hline Peak power $[\mathrm{MW} / \mathrm{m}]$ & 0.2 & 31 & 230 \\
\hline \# of klystrons & 616 & 3312 & - \\
\hline Pulse length $[\mathrm{ns}]$ & $9 \cdot 10^{5}$ & 224 & 100 \\
\hline Rep. Rate $[\mathrm{Hz}]$ & 5 & 120 & 200 \\
\hline Bunch spacing $[\mathrm{ns}]$ & 337 & 2.8 & 0.67 \\
\hline \# of bunches pp & 2820 & 95 & 150 \\
\hline Bunch charge $\left[10^{10} \mathrm{e}\right]$ & 2 & 0.95 & 0.6 \\
\hline
\end{tabular}

Complete conceptual designs for the X-band [6,7] and TESLA [8] colliders have been worked out, including all sub-systems such as Final Focus, injection systems, etc. The layout of the sub-systems takes the possibility of energy upgrade into account. For TESLA $\mathrm{E}_{\mathrm{cm}}=800 \mathrm{GeV}$ can be reached within the foreseen $32 \mathrm{~km}$ site with improved performance of the s.c. cavities at $\mathrm{g}=34$ $\mathrm{MV} / \mathrm{m}$. For X-band, the $30 \mathrm{~km}$ site length accommodates an upgrade to $1 \mathrm{TeV}$, eventually extendable to $1.5 \mathrm{TeV}$.

The CLIC group has recently been focusing much attention on the two-beam linac layout and parameters in the very high energy regime, $3-5 \mathrm{TeV}$ [9].

As part of the TESLA project study [8], the integration of a coherent (FEL) X-ray user facility in the Angstroem wavelength regime is foreseen. DESY as the coordinating laboratory of the TESLA collaboration has taken over the charge to investigate a site next to DESY suitable for the integrated Linear Collider/Free Electron Laser facility. A detailed study of the required civil construction work and the preparations for the legal procedure necessary for project approval are in progress.

\section{TEST FACILITIES}

The development of the technology required for the next generation linear collider is pursued in the framework of test facilities at several laboratories, see Table 2. The SLC as the only existing linear collider is included in this list. The experience gained from years of successful operation of the SLC in many fields of accelerator physics and technology has proven invaluable for the development of future linear collider projects. Furthermore, the $50 \mathrm{GeV}$ SLC linac has been used to test a beam optical system (Final Focus system) required to provide de-magnification of the spot size to the tiny values foreseen for the next generation machine.

Table 2: Overview of linear collider test facilities

\begin{tabular}{|l|c|l|}
\hline SLC & Only existing L.C. & SLAC \\
\hline \hline FFTB & Final Focus System & SLAC/int. coll. \\
\hline NLC-TA & 400 MeV X-band linac & SLAC \\
\hline ASSET & $\begin{array}{c}\text { structure test } \\
\text { (wakefields, HOM) }\end{array}$ & SLAC \\
\hline ATF & $\begin{array}{c}1.54 \text { GeV low- } \varepsilon \\
\text { damping ring }\end{array}$ & KEK \\
\hline TTF & $\begin{array}{c}\text { s.c. cavity } \\
\text { development, } 500 \\
\text { MeV linac }\end{array}$ & DESY/int. coll. \\
\hline CTF & two-beam test acc. & CERN \\
\hline
\end{tabular}

\subsection{FFTB}

The Final Focus Test Beam experiment, set up at the end of the SLAC two-mile linac, was constructed in international collaboration of institutes from 6 countries

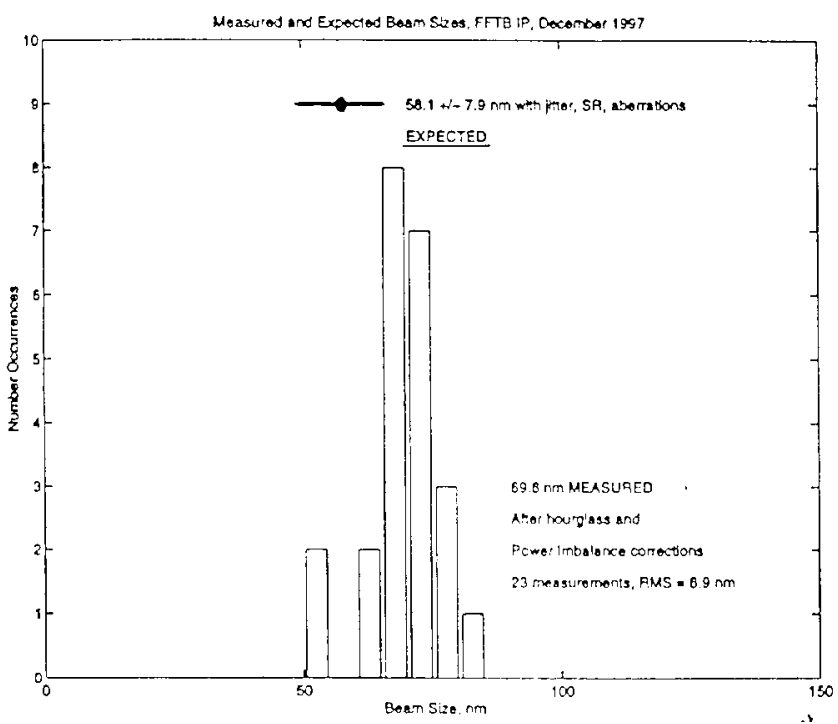

Figure 1: Vertical spot size measurements at the FFTB in comparison with the expected value $(58 \pm 8 \mathrm{~nm})$.

led by SLAC. It comprises a complete beam line very similar to the system required for a future machine, except, of course, for the lower beam energy. First successful operation of the FFTB took place in 1994 [10]. 
Evaluation of measurements done in Dec. 1997 yield a vertical spot size of about $70 \mathrm{~nm}$ in reasonable good agreement with the expected value (see Fig. 1). In the analysis, a measured orbit jitter of $40 \mathrm{~nm}$ was taken into account.

\subsection{NLCTA, ASSET}

The X-band linac technology is under development at SLAC in collaboration with KEK. The $400 \mathrm{MeV}$ test linac of NLCTA [11] represents a full integrated system test of of the components for the X-band collider. Beam acceleration with a maximum gradient of $40 \mathrm{MV} / \mathrm{m}$ was achieved at $50 \%$ of the design intensity $(600 \mathrm{~mA})$ and reduction of the multi-bunch energy spread to $0.3 \%$ peakto-peak with beam loading compensation was successfully demonstrated. High power klystrons have been developed with periodic permanent magnet (ppm) focusing (Fig. 2), avoiding the considerable power consumption otherwise due to the focusing solenoid. With a peak power of $50 \mathrm{MW}$ at $2 \mu \mathrm{s}$ pulse length and an efficiency of $55 \%$ all specifications have been met. Work on a 75MW ppm focused klystron is in progress both at SLAC and KEK. RF-pulse compression by more than a factor of three was achieved with a SLED-II system. The application of a delay line distribution system for pulse compression is foreseen for the future [12,13]. It is also planned to replace the present conventional modulator concept by a more efficient and lower cost induction type device based on solid state technology.

Several $1.8 \mathrm{~m}$ long X-band structures have been built and tested. The concept of detuning and damping of higher order modes (HOM) is applied to provide a sufficiently strong suppression of long-range wakefields. Beam tests of X-band structures at ASSET [14] (see Fig. 3) have demonstrated the wakefield suppression as well as the possibility to use the HOM signal for precise beam-based alignment of the structures as required for beam stability. Furthermore, the challenge of obtaining a structure straightness at the $10 \mu \mathrm{m}$ level has been addressed [15].

\subsection{ATF}

The accelerator test facility at KEK comprises a $1.54 \mathrm{GeV}$ electron beam injector linac and damping ring and represents an (almost) full size model of a low emittance injection system for the X-band linear collider. So far, operation took place at $1.29 \mathrm{GeV}$ and with damping wigglers off, leading to a damping time of $30 \mathrm{~ms}$ as compared to the design value of $9 \mathrm{~ms}$. With single bunch operation at $6-8 \cdot 10^{9}$ bunch charge, the measured emittances are approaching the ambitious design goals of $3 \cdot 10^{-6} \mathrm{~m}$ (horizontal, normalised) and $3 \cdot 10^{-8} \mathrm{~m}$ (vertical, norm.) [16].

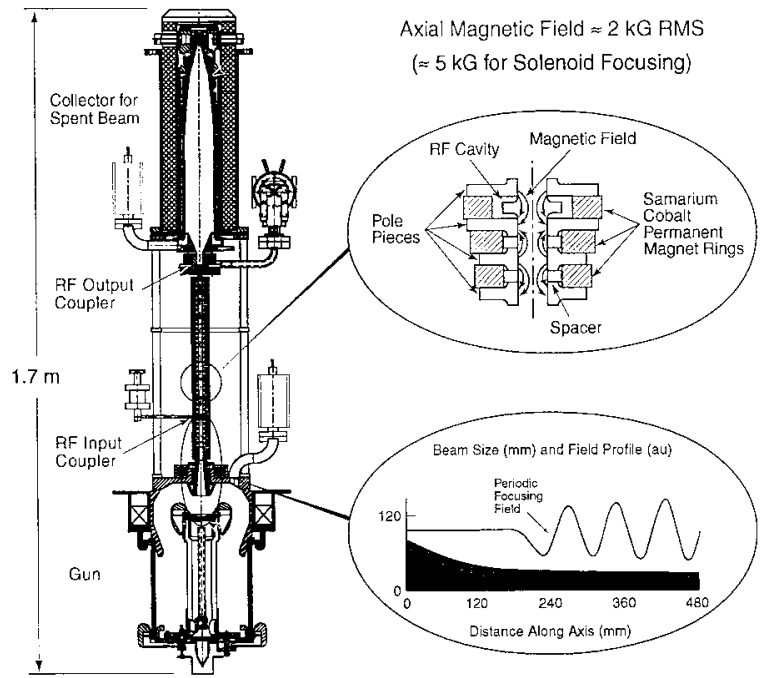

Figure 2: The ppm focused X-band klystron developed at SLAC.
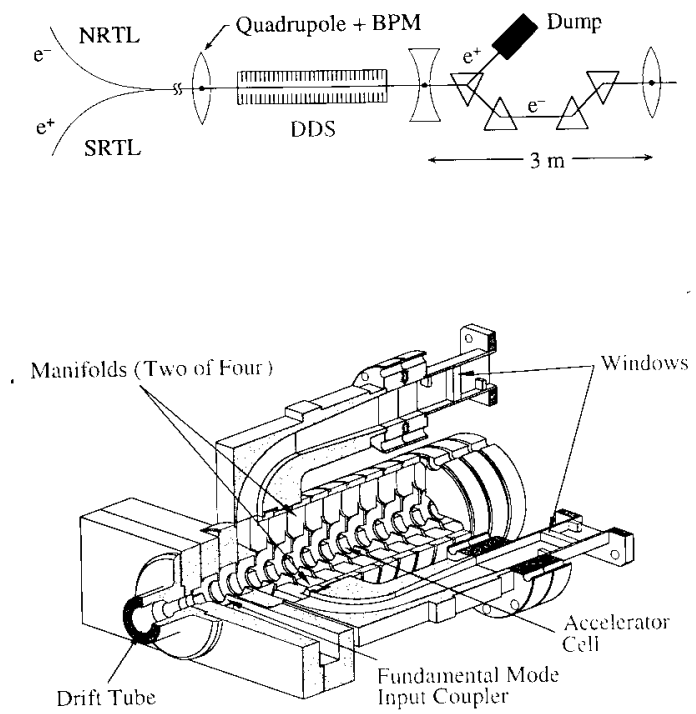

Figure 3: Sketch of the accelerating structure set up (top) at the SLC linac and of the X-band damped-detuned structure (bottom).

\section{$3.4 C T F$}

The main objective of the CLIC test facility at CERN is the demonstration of the two-beam acceleration concept at a frequency of $30 \mathrm{GHz}$. The CTF drive beam line uses a high intensity RF-laser gun, S-band accelerating section and bunch compressor. In 1998 a bunch train with total charge up to $450 \mathrm{nC}$ was sent through the $30 \mathrm{GHz}$ decelerating/power transfer structure, generating up to $50 \mathrm{MW}$ of RF-power $[9,17]$. The maximum field produced in the main beam accelerating structure was $69 \mathrm{MV} / \mathrm{m}$. First test of an active alignment system for the linac components achieved a position stability of $2 \mu \mathrm{m}$ (rms). 
For the future, it is planned to upgrade the CTF for a full system test of the newly developed drive beam generation scheme $[18,19]$, as it would be used in a multi-TeV twobeam linear collider facility.

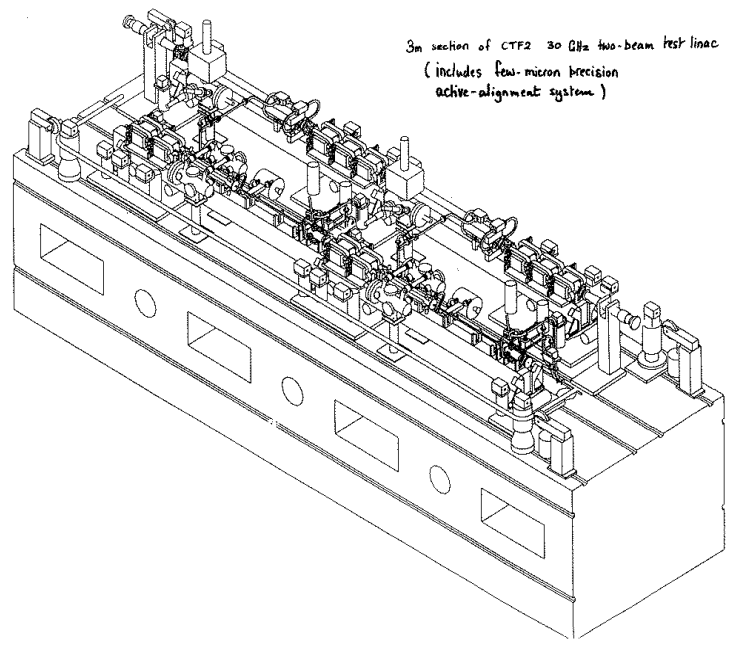

Figure 4: One section of the two-beam accelerator at CTF.

\section{$3.5 \mathrm{TTF}$}

The development of high-performance 9-cell superconducting Niobium cavities (Fig. 5) is pursued by the international TESLA collaboration (involving more than 30 institutes from 9 countries) in the framework of the TESLA test facility at DESY.

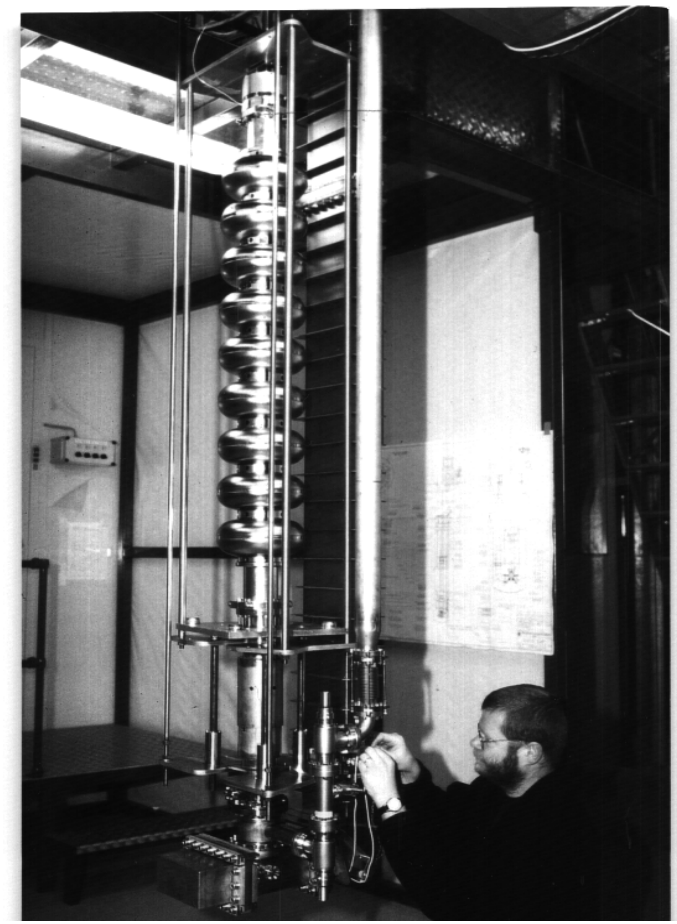

Figure 5: One of the TESLA Niobium resonators being prepared for CW-RF test in the vertical test stand.
The TTF includes the complete infrastructure for cavity processing (clean rooms, chemical and high-temperature treatment, high-pressure rinsing with ultra-pure water), vertical and horizontal test stands for CW- and pulsed-RF operation of the $1.3 \mathrm{GHz}$ resonators and a $500 \mathrm{MeV}$ linac for a full integrated system test of the accelerator modules with beam. The evolution of the achieved accelerating gradients in vertical test over the last years is shown in Fig. 6. High gradients above $20 \mathrm{MV} / \mathrm{m}$ were reached already at an early stage, but the failure rate (cavities with poor performance due to fabrication errors) was rather high at that time. The results from the more recent production series shows a strong improvement in the performance statistics, so that the gradient of $22 \mathrm{MV} / \mathrm{m}$ required for the $500 \mathrm{GeV}$ collider is now achieved on average (see ref. [20] for more details). Cavities equipped with RF- and HOM-couplers underwent pulsed power tests in the horizontal test stand. No systematic degradation of performancecompared to the vertical test was observed (Fig. 7).

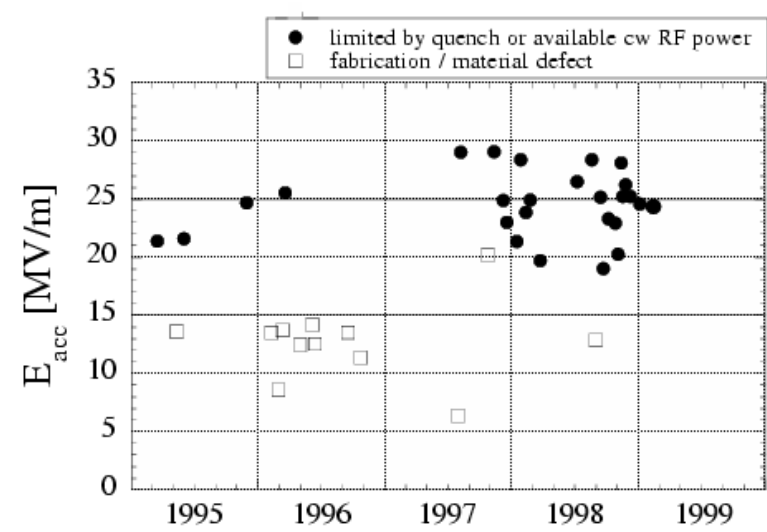

Figure 6: Evolution of accelerating gradient achieved with TESLA cavities on the vertical test stand.

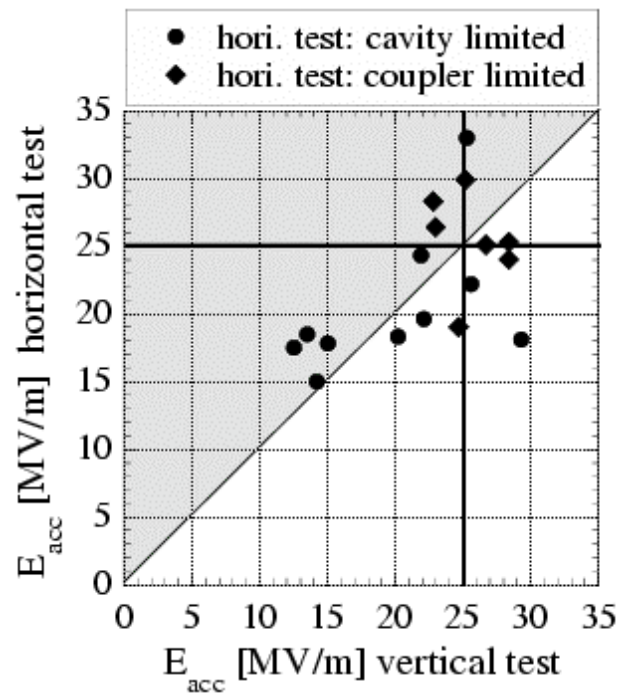

Figure 7: Horizontal vs. vertical test stand results. 
Eight 9-cell cavities are assembled into a string and mounted in an accelerator module, which also contains focusing and beam diagnostics devices. So far two of these modules have been installed in the TTF linac and tested with beam. The $1^{\text {st }}$ module reached a gradient of $16 \mathrm{MV} / \mathrm{m}$ in May 1997 . The $2^{\text {nd }}$ module was tested early this year and showed a gradient of $18.5 \mathrm{MV} / \mathrm{m}$ measured with beam and $20.8 \mathrm{MV} / \mathrm{m}$ obtained from RFmeasurement, in both cases limited by insufficient RFpower coupler conditioning. From cryogenic measurements a resonator quality factor of $1.3 \cdot 10^{10}$ at $\mathrm{T}=2 \mathrm{~K}$, exceeding the TESLA design goal of $10^{10}$, could be deduced. A $3^{\text {rd }}$ module is presently being installed, replacing module \#1, and expected (from test stand results for the individual resonators) to yield an average gradient of $25 \mathrm{MV} / \mathrm{m}$. The TTF beam line is also being prepared for a first test of the SASE FEL concept, which will take place after beam operation resumes in summer this year.

Accelerating gradients above $30 \mathrm{MV} / \mathrm{m}$ have already been achieved with some of TESLA cavities (the record value being $33 \mathrm{MV} / \mathrm{m}$ at full pulse length and rep. rate in the horizontal test stand). The TESLA cavity R\&D will continue to define a reliable procedure, suitable for mass production, for obtaining an average gradient of $34 \mathrm{MV} / \mathrm{m}$ as required for the energy upgrade to $800 \mathrm{GeV}$. One very promising improvement of resonator performance was recently obtained by electro-polishing [21], with gradients up to $37 \mathrm{MV} / \mathrm{m}$ obtained in single cell L-band cavities.

\section{CONCLUSIONS}

The technical developments towards a next generation linear collider are far advanced and the X-band and TESLA groups will present technical proposals, incuding cost estimates and schedule, within the next two years.

The recent progress of the CLIC group on both the test facility and the conceptual design of the two-beam linear collider is remarkable. These developments, to my mind, should be seen on a longer time scale. At present the twobeam approach appears to be the most promising concept for a multi-TeV lepton collider, which may be realised as a facility following the next generation linear collider.

Being left with two concepts for the next machine, the obvious question is which one to choose. This decision will have to involve a detailed comparison of the scientific potential as well as the cost of the X-band and TESLA facilities.

\section{ACKNOWLEDGEMENT}

I would like to express my thanks to my colleagues in the linear collider design groups for providing information and material to prepare this talk, especially to J.-P. Delahaye, T. Raubenheimer and N. Toge.

\section{REFERENCES}

[1] G. A. Loew (ed.), International Linear Collider Technical Review Committee Report, SLAC-R-471, 1995.

[2] I. Reyzl and R.-D. Kohaupt, contribution to this conference TUA41.

[3] T. O. Raubenheimer, contribution to this conference TUDR1.

[4] K. Kubo, T. Higo and K. Yokoya, contribution to this conference FRA22.

[5] S. Fartoukh, A. Mosnier and O. Napoly, contribution to this conference FRA10.

[6] NLC Zeroth Order Design Report, SLAC-R-474, 1996.

[7] JLC Design Study, KEK 1997.

[8] R. Brinkmann, G. Materlik, J. Roßbach and A. Wagner, Conceptual Design of a $500 \mathrm{GeV}$ e+e- Linear Collider with Integrated X-ray Laser Facility, DESY-1997-048.

[9] J.-P. Delahaye et al., contribution to this conference TUDR3.

[10] D. Burke et al., Proc. $4^{\text {th }}$ EPAC London 1994, Vol. I, p. 23.

[11] C. Adolphsen et al., Proc. $17^{\text {th }}$ PAC Vancouver 1997, Vol. I, p. 439.

[12] S. G. Tantawi, R. D. Ruth and P. B. Wilson, contribution to this conference THAL3.

[13] Y. H. Chin et al., contribution to this conference FRA15.

[14] M. Seidel, Proc. 17 $7^{\text {th }}$ PAC Vancouver 1997, Vol. I, p. 434.

[15] T. Higo et al., contribution to this conference FRA16.

[16] T. Okugi et al., contribution to this conference TUDR5.

[17] R. Bossart et al., CLIC note 382, CERN 1998.

[18] R. D. Ruth, Proc. $6^{\text {th }}$ EPAC Stockholm 1998, Vol. I, p. 248.

[19] R. Corsini, contribution to this conference FRA17.

[20] M. Pekeler, contribution to this conference TUDR2.

[21] E. Kako et al., contribution to this conference THAL6. 